\title{
Pretreatment with angiotensin-converting enzyme inhibitor improves doxorubicin-induced cardiomyopathy via preservation of mitochondrial function
}

\author{
Asimina Hiona, PhD, ${ }^{\text {a }}$ Andrew Stephen Lee, BS, ${ }^{a}$ Jayan Nagendran, $\mathrm{MD}, \mathrm{PhD},{ }^{\mathrm{b}}$ Xiaoyan Xie, PhD, ${ }^{\mathrm{a}}$ \\ Andrew J. Connolly, MD, PhD, ${ }^{\mathrm{c}}$ Robert C. Robbins, $\mathrm{MD},{ }^{\mathrm{b}}$ and Joseph C. Wu, MD, PhD ${ }^{\mathrm{a}, \mathrm{d}}$
}

\begin{abstract}
Objective: Doxorubicin is a widely used chemotherapy drug, but its application is associated with cardiotoxicity. Free radical generation and mitochondrial dysfunction are thought to contribute to doxorubicin-induced cardiac failure. Angiotensin-converting enzyme inhibitors are commonly used as cardioprotective agents and have recently been shown in clinical studies to be efficacious in the prevention of anthracycline-induced heart failure. This study evaluated a mechanism for these protective effects by testing the ability of the angiotensinconverting enzyme inhibitor enalapril to preserve mitochondrial function in a model of chronic doxorubicin treatment in rats.
\end{abstract}

\begin{abstract}
Methods: Sprague Dawley rats were divided into 3 groups and followed for a total of 10 weeks: (1) controluntreated, (2) doxorubicin treated, and (3) doxorubicin + enalapril treated. Doxorubicin was administered via intraperitoneal injection at weekly intervals from weeks 2 to 7 . Enalapril was administered in the drinking water of the doxorubicin + enalapril group for the study duration.
\end{abstract}

Results: Doxorubicin treatment produced a significant loss in left ventricular contractility $(P<.05)$, decrease in mitochondrial function via impairment of state-3 respiration, decrease in the cytosolic fraction of adenosine triphosphate, and up-regulation of free radical production. Enalapril significantly attenuated the decrease in percent fractional shortening $(P<.05)$ and prevented the doxorubicin-associated reduction in respiratory efficiency and cytosolic adenosine triphosphate content $(P<.05)$. Enalapril also abolished the robust doxorubicin-induced increase in free radical formation.

Conclusions: Administration of enalapril attenuates doxorubicin-induced cardiac dysfunction via preservation of mitochondrial respiratory efficiency and reduction in doxorubicin-associated free radical generation. (J Thorac Cardiovasc Surg 2011;142:396-403)

Supplemental material is available online.

Doxorubicin, an anthracycline, is a widely used cytotoxic agent for the treatment of human neoplasms, such as leukemias and Hodgkin's lymphoma. However, administration of doxorubicin is known to cause cardiomyopathy, eventually

From the Division of Cardiology, ${ }^{\mathrm{a}}$ Department of Medicine, and Departments of Cardiothoracic Surgery, ${ }^{\mathrm{b}}$ Pathology, ${ }^{\mathrm{c}}$ and Radiology, ${ }^{\mathrm{d}}$ Stanford University School of Medicine, Stanford, Calif.

This work was supported in part by grants from the Burroughs Wellcome Fund's Career Awards for Medical Scientists, CA BCRP 14IB-0039 (to J.C.W.), National Institutes of Health HL 095571 and HL 089027 (to J.C.W.), and Howard Hughes Medical Institute Fellowship (to A.S.L.).

Disclosures: Authors have nothing to disclose with regard to commercial support.

A.H. and A.S.L. contributed equally to the preparation of this manuscript.

Received for publication Feb 9, 2010; revisions received May 30, 2010; accepted for publication July 5, 2010; available ahead of print Nov 22, 2010.

Address for reprints: Joseph C. Wu, MD, PhD, Stanford University School of Medicine, 300 Pasteur Drive, Grant S140, Stanford, CA 94305-5344 (E-mail: joewu@ stanford.edu).

$0022-5223 / \$ 36.00$

Copyright (๑) 2011 by The American Association for Thoracic Surgery doi:10.1016/j.jtcvs.2010.07.097 leading to congestive heart failure that almost invariably develops in patients receiving cumulative doses of doxorubicin more than $550 \mathrm{mg} / \mathrm{m}^{2} .{ }^{1}$ As a result of doxorubicin therapy, a growing number of patients in the United States survive childhood and adult cancers only to develop severe cardiac dysfunction after treatment. ${ }^{2}$ These patients have few surgical options for treatment because they have a combination of both dilated and restrictive cardiomyopathies, and make poor heart transplant candidates because of their history of cancer. Development of medical therapies that aid in the management of cardiac dysfunction is thus paramount for the long-term survival of these individuals.

Several previous studies have implicated free radical formation and oxidative stress as central mechanisms underlying the cardiotoxic effects of doxorubicin. ${ }^{3}$ As a result, many experimental drug treatments to ameliorate cardiotoxicity of doxorubicin have focused on the protective effects of antioxidants and metal chelators, which minimize free radical damage. ${ }^{4}$ Although these substances have shown some benefit, they are difficult to administer because of the challenge of achieving constant plasma concentrations of antioxidant drugs and their poor uptake by the heart. $^{5}$ 


\author{
Abbreviations and Acronyms \\ $\mathrm{ACE}=$ angiotensin-converting enzyme \\ $\mathrm{ADP}=$ adenosine diphosphate \\ ATP $=$ adenosine triphosphate \\ $\mathrm{ETC}=$ electron transport chain \\ $\mathrm{FRL}=$ free radical leak \\ $\mathrm{NS}=$ not significant \\ $\mathrm{P} / \mathrm{M}=$ pyruvate/malate \\ $\mathrm{RCR}=$ respiratory control ratio \\ ROS $=$ reactive oxygen species
}

Angiotensin-converting enzyme (ACE) inhibitors are a class of drugs that are routinely administered in the clinic and have clearly shown positive therapeutic profiles for the treatment of heart failure caused by a number of cardiovascular diseases. ${ }^{6}$ ACE inhibitors possess free radical scavenger and antioxidant properties, ${ }^{7}$ and have recently been shown in 2 prospective clinical studies to be efficacious in the prevention of anthracycline-induced heart failure when administered early after chemotherapy regimens. ${ }^{8,9}$ Administration of ACE inhibitors in these trials and preclinical studies has not been linked with an increased rate of recurrent malignancy. ${ }^{1,10}$ However, only a limited number of studies have investigated the mechanisms by which ACE inhibition can prevent anthracycline-induced cardiotoxicity. ${ }^{7,11}$ The potential effects ACE inhibitors may have on prevention of anthracycline-induced mitochondrial dysfunction remain unknown. To answer these questions, this study aimed to (1) investigate in vivo the possible protective effects of the ACE inhibitors (enalapril) against doxorubicin-induced cardiac toxicity in a longitudinal model of treatment and (2) determine whether the improved cardiac function from ACE inhibitor therapy is due to improved mitochondrial function and reduced free radical generation. Our results show that the concurrent administration of ACE inhibitors with doxorubicin treatment not only ameliorates cytotoxic effects of doxorubicin but also prevents doxorubicin-induced free radical formation and preserves mitochondrial respiratory efficiency and cellular adenosine triphosphate (ATP) content.

\section{MATERIALS AND METHODS}

\section{Animals}

Twenty-four female Sprague Dawley rats at approximately 10 weeks of age were obtained from Charles River Laboratories (Wilmington, Mass). The animals were divided into 3 groups: a) control-untreated $(\mathrm{n}=8), \mathrm{b})$ doxorubicin treated (Dox) $(\mathrm{n}=8)$, and c) doxorubicin + enalapril treated $(\mathrm{DE})(\mathrm{n}=8)$. Both Dox and $\mathrm{DE}$ groups received doxorubicin at a cumulative dose of $25 \mathrm{mg} / \mathrm{kg}$ administered weekly via intraperitoneal injection for 6 weeks. Enalapril pretreatment for the DE group was started 1 week before administration of the first doxorubicin injection and continued throughout the study and for an additional 3 weeks after the last doxorubicin injection (Figure 1,A). The dosage of enalapril was calculated on the basis of a previous study by Sanbe and colleagues ${ }^{12}$ and set at a dose of $10 \mathrm{mg} / \mathrm{kg} / \mathrm{d}$ for each animal as previously described. ${ }^{12}$ A detailed description is included in the Supplemental Materials and Methods.

\section{Assessment of Cardiac Function}

Echocardiography studies were performed before treatment and immediately before animal sacrifice to determine left ventricular fractional shortening, using a 14.7-MHz transducer on a Sequoia C512 echocardiography system (Siemens, Malvern, Pa). A detailed description is included in the Supplemental Materials and Methods.

\section{Tissue Histology}

Heart and liver specimens were fixed in $4 \%$ paraformaldehyde and stained with hematoxylin-eosin for analysis by light microscopy. A detailed description is included in the Supplemental Materials and Methods.

\section{Enzymatic Measurement of Caspase-3 and Caspase-9 Activities}

Caspase-3 and caspase- 9 activities were measured using fluorometric protease assay kits: Caspase-3/CPP32 and Caspase-9/Mch6 (Biovision, Mountain View, Calif). A complete description is included in the Supplemental Materials and Methods.

\section{Mitochondrial and Cytosolic Isolation}

Mitochondrial and cytosolic protein fractions were isolated using differential centrifugation as previously described. A detailed description is included in the Supplemental Materials and Methods.

\section{Mitochondrial Respiration and $\mathrm{H}_{2} \mathrm{O}_{2}$ Generation}

Mitochondrial oxygen consumption was measured at $37^{\circ} \mathrm{C}$ by polarography, with a Clark-type oxygen electrode (Oxytherm, Hansatech, Norfolk UK) under identical conditions (same mitochondria, buffer composition, and substrate concentrations) to $\mathrm{H}_{2} \mathrm{O}_{2}$ production measurements. The rate of mitochondrial $\mathrm{H}_{2} \mathrm{O}_{2}$ production was assayed in freshly isolated mitochondria by a fluorometric method described by Barja. ${ }^{13}$ A complete description is included in the Supplemental Materials and Methods.

\section{Adenosine Triphosphate Content}

Cytosolic homogenate isolated from heart was used immediately after isolation to determine ATP content using a bioluminescence ATP kit from Sigma-Aldrich Inc (St Louis, Mo). A complete description is included in the Supplemental Materials and Methods.

\section{Statistical Analysis}

Data were analyzed using a 1-way analysis of variance with NewmanKeuls post hoc comparison. All data are represented as average \pm standard error of the mean.

\section{RESULTS}

Rats were studied longitudinally over 10 weeks (Figure 1, A). Control animals were compared with the Dox and DE groups. There was a $100 \%$ survival in both control and DE groups up to 10 weeks. In contrast, the Dox group experienced only a $75 \%$ survival ( 2 deaths) (Figure $1, B$ ). These data show that a 10 -week model of doxorubicin administration causes significant toxicity resulting in mortality and validates the time course for intervention.

Doxorubicin treatment was observed to cause a significant decrease in cardiac function, as quantified by depressed 


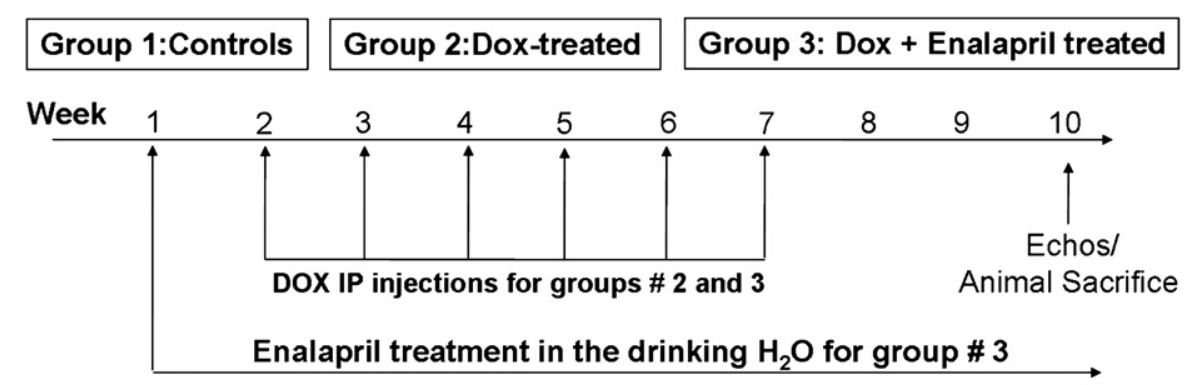

Dox Cumulative Dose: $25 \mathrm{mg} / \mathrm{kg}$, administered once a week for 6 weeks.

A Enalapril Dose: $10 \mathrm{mg} /$ week, administered every day throughout the study.

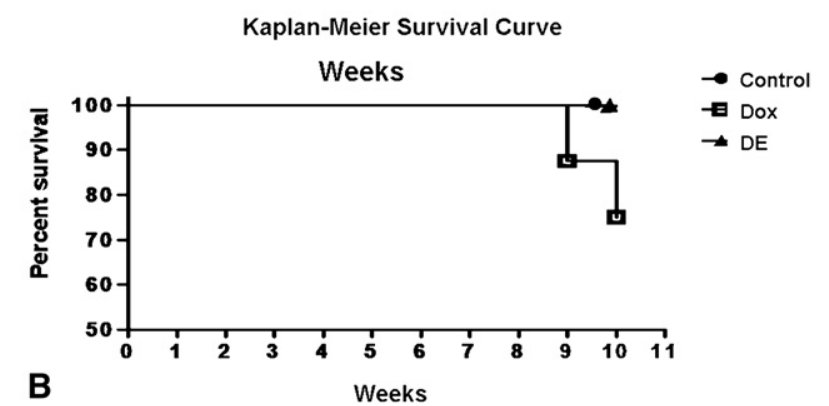

FIGURE 1. Study design and animal survival. A, Schematic for study design. B, Kaplan-Meier survival curve for control, Dox, and DE animals during the duration of the study. $P=$ NS. Dox, Doxorubicin; $I P$, intraperitoneal; $D E$, doxorubicin + enalapril.

fractional shortening of the left ventricle via echocardiography (baseline before Dox treatment: $38.62 \% \pm 0.77 \%$, post-treatment control: $39.28 \% \pm 0.83 \%$ vs Dox: $24.92 \%$ $\pm 3.08 \%, P<.05)$. Enalapril treatment significantly attenuated loss of systolic function in the DE group (DE: $31.42 \%$ $\pm 1.06 \%, P<.05$ vs Dox) (Figure 2, $A, B$ ). These findings confirm previous findings, including 2 recent human clinical studies showing that simultaneous administration of enalapril can significantly blunt the deleterious cardiotoxic effects of doxorubicin on heart function. ${ }^{7-9,14}$

Doxorubicin not only reduced contractility in treated animals but also caused pathologic changes as observed by histology. Histologic sections of the heart in Dox animals showed numerous scattered foci of myofibrillar degeneration with associated contraction bands (Figure 2,C). By comparison, DE hearts showed only elongated cardiomyocytes. The livers in both Dox and DE groups showed gross enlargement, thickened and inflamed capsules, indications of venous congestion, and hepatocyte cytopathology with regenerative changes.

The decline in cardiac function and histologic changes of the myocardium caused by doxorubicin are not fully understood. Apoptosis has been implicated in doxorubicininduced cardiac dysfunction. ${ }^{15}$ We found that apoptosis levels as measured by caspase- 3 activity in the cytosol of Dox and DE rat hearts were significantly higher than those in control animals (control: $122.2 \pm 8.7 \mathrm{FU} / \mathrm{mg}$ protein vs
Dox: $165.5 \pm 19.8 \mathrm{FU} / \mathrm{mg}$ protein vs DE: $169.0 \pm 9.2$ FU/mg protein, $P<.05$ ). Caspase-9 activity was also elevated in Dox and DE rats compared with controls (control: $196.4 \pm 15.1 \mathrm{FU} / \mathrm{mg}$ protein vs Dox: $251.6 \pm 11.8 \mathrm{FU} / \mathrm{mg}$ protein vs DE: $252.9 \pm 16.0 \mathrm{FU} / \mathrm{mg}$ protein, $P<.05$ ) (Figure 3, A). Comparison of caspase-3 activity with that of caspase-9 revealed a significant positive correlation $(r=0.65, P<.01$ ) (Figure $3, B)$, providing evidence for induction of the mitochondrial-mediated pathway of apoptosis. These data corroborate previous findings that apoptosis levels are increased in the myocardium by doxorubicin and show that the increase in cardiac apoptosis is not significantly attenuated by enalapril.

Because enalapril was observed to improve systolic function after doxorubicin treatment but did not prevent apoptosis, we sought to determine whether protection against anthracycline-induced toxicity by ACE inhibitors was due to an alternative mechanism. Several recent clinical studies have suggested that ACE inhibition may be efficacious in the prevention of anthracycline-induced heart failure by protection of mitochondrial function. ${ }^{8,9}$ However, these reports did not show causality between antioxidant properties of enalapril and improvement in systolic function in patients post-chemotherapy. To test whether doxorubicin significantly affects mitochondrial processes and enalapril can ameliorate these changes, we evaluated oxygen consumption in intact mitochondria isolated from the hearts of 

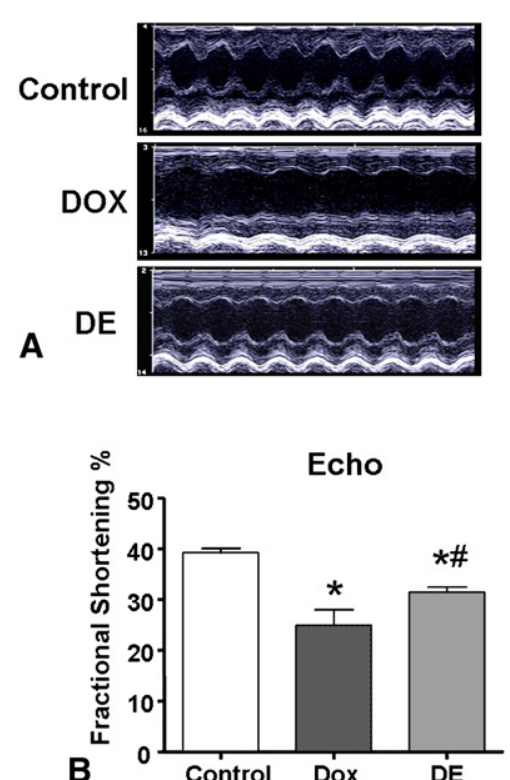

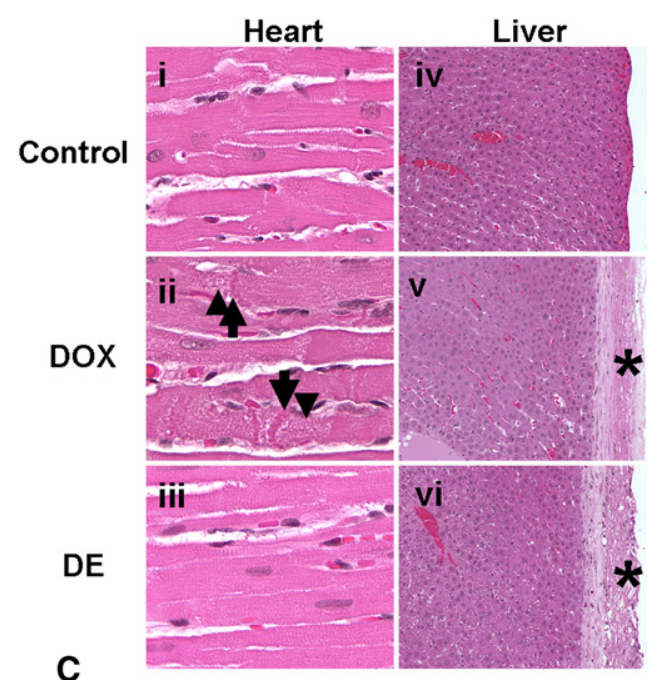

FIGURE 2. Doxorubicin treatment results in a significant loss of heart function and myofibrillar degeneration in rats, whereas enalapril significantly attenuates these changes. A, Left ventricular fractional shortening was measured by echocardiography before animal sacrifice. Representative echocardiograms are shown for control, Dox, and DE animals 10 weeks after infarction. B, Statistical analysis of echocardiographic measures. Error bars represent the standard error of the mean (SEM). *,\#P<.05. *Significantly different compared with Dox and DE groups. *Significantly different compared with control and DE groups. C, Enalapril treatment prevents significant myofibrillar degeneration with contraction band formation in doxorubicin-treated hearts but does not prevent liver cytopathic changes. Compared with control hearts $(i)$, Dox hearts $(\mathrm{n}=3)($ ii) exhibited numerous contraction bands $($ arrows) with nearby myofibrillar degeneration (arrowheads), whereas only myocyte elongation is seen in the DE heart (iii). Compared with control livers (iv), the Dox and DE livers ( $v$ and $v i$ ) showed capsular fibrosis and inflammation [asterisks], hepatocyte swelling, and pleiomorphic cords of hepatocytes. DOX, Doxorubicin; $D E$, doxorubicin + enalapril.

Dox, DE, and control animals. Addition of pyruvate/malate (P/M) was used to simulate state 4 , the inactive state of mitochondria, and addition of $\mathrm{P} / \mathrm{M}$ with adenosine diphosphate (ADP) as substrates was used to simulate state 3, the active state of mitochondria. ${ }^{16}$

Treatment with doxorubicin alone (Dox group) decreased oxygen consumption levels in both states 3 and 4 (Figure 4, A). This effect was particularly pronounced in state 3 , the ATP producing a state of mitochondria where oxygen consumption typically increases robustly. Although doxorubicin alone decreased mitochondrial oxygen consumption by 2.5 -fold in state 3 compared with control animals, the addition of enalapril (DE group) completely reversed this effect (control: $47.66 \pm 4.15$ nmoles $\mathrm{O}_{2} / \mathrm{min} \mathrm{mg}$ protein, Dox: $18.94 \pm 4.67$ nmoles $\mathrm{O}_{2} / \mathrm{min} \mathrm{mg}$ protein, DE: $50.44 \pm 6.90$ nmoles $\mathrm{O}_{2} / \mathrm{min} \mathrm{mg}$ protein, $P<.05$ for control vs Dox and DE vs Dox, $P=$ not significant $[\mathrm{NS}]$ for control vs DE) (Figure 4, $A$ ). Doxorubicin also significantly lowered oxygen consumption in state 4 mitochondria, the inactive state of mitochondria in which ADP phosphorylation does not occur. As with state 3 , enalapril completely abolished this effect. These findings suggest that enalapril can prevent mitochondrial dysfunction caused by doxorubicin toxicity by allowing mitochondrial state 3 and state 4 respiration to be maintained at control levels.

Correlation of active to inactive mitochondrial coupling was further determined by calculating the respiratory control ratio (RCR), defined as state 3 oxygen consumption/ state 4 oxygen consumption. By calculating the RCR, we found that doxorubicin not only decreased inactive (state 4) and active (state 3 ) mitochondrial respiration but also lowered the functional coupling of oxygen consumption at baseline (state 4) with the efficiency of energy production by ADP phosphorylation (state 3 ). Addition of enalapril significantly ameliorated the disproportional decrease in RCR and impairment of mitochondrial metabolic activity induced by doxorubicin (control RCR: $6.747 \pm 0.428$, Dox RCR: $3.666 \pm 0.791$, DE RCR: $5.458 \pm 0.516, P<.05$ for control vs Dox, $P=\mathrm{NS}$ for DE vs control) (Figure $4, A$ ).

To test whether impairment of oxidative respiration affected mitochondrial output, we next evaluated ATP production in the hearts of animals treated with doxorubicin. ATP produced by mitochondria is exported into the cytosol for cellular function. ATP content was therefore measured in the cytosolic homogenate of heart tissue from animals in the control, Dox, and DE groups. Total ATP content in the cytosolic homogenate was found to be significantly 

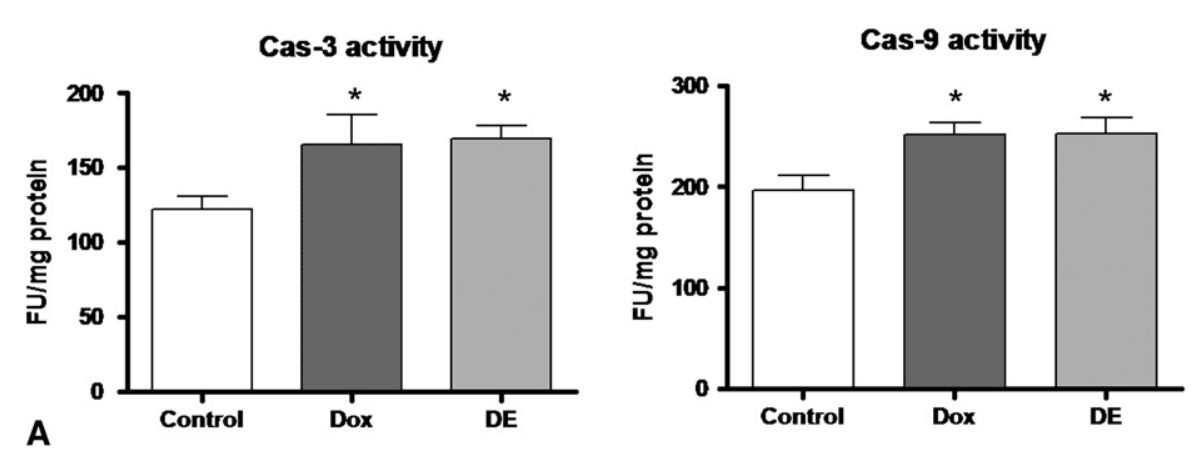

Pearson Correlation

depleted in doxorubicin-treated rats compared with control animals (control: $27.87 \pm 2.37$ fmoles ATP/mg protein, Dox: $14.44 \pm 3.92$ fmoles ATP/mg protein, $P<.05$ ) (Figure $4, B$ ). The addition of enalapril completely reversed this effect, increasing the cytosolic ATP content to near control levels in the DE group of animals (control: $27.87 \pm 2.37$ fmoles ATP/mg protein, DE: $24.24 \pm 1.66$ fmoles ATP $/ \mathrm{mg}$ protein, $P=\mathrm{NS}$ ). This outcome suggests that the impairment of mitochondrial respiratory efficiency by doxorubicin results in depletion of ATP for cellular use, and that enalapril is able to reverse this effect by preserving normal levels of oxidative respiration.

Finally, because several previous studies have shown that dysfunction of mitochondrial respiration and production of ATP are linked to increased generation of reactive oxygen species (ROS) ${ }^{17}$ we assessed levels of oxidative stress in the hearts of control, Dox, and DE animals. ROS production was measured as the amount of $\mathrm{H}_{2} \mathrm{O}_{2}$ released from the mitochondria in an incubation medium supplemented with $\mathrm{P} / \mathrm{M}$ to evaluate total basal mitochondrial ROS generation. ${ }^{13}$ Absolute values for basal levels of $\mathrm{H}_{2} \mathrm{O}_{2}$ production were not significantly different among control, Dox, and DE animals (control: $0.313 \pm 0.028$ nmoles $\mathrm{H}_{2} \mathrm{O}_{2} / \mathrm{min}$ mg protein vs Dox: $0.345 \pm 0.039$ nmoles $\mathrm{H}_{2} \mathrm{O}_{2} /$ min mg protein vs
DE: $0.353 \pm 0.037$ nmoles $\mathrm{H}_{2} \mathrm{O}_{2} /$ min mg protein, $P=\mathrm{NS}$ ) (Figure 5, A). Although these results may seem to suggest that doxorubicin administration is not associated with increased oxidative stress, these findings actually indicate the opposite - that free radical generation is elevated in the hearts of animals treated with doxorubicin. This is due to the consideration that $\mathrm{H}_{2} \mathrm{O}_{2}$ generation is typically proportional to mitochondrial $\mathrm{O}_{2}$ consumption. ${ }^{20}$ Because levels of $\mathrm{O}_{2}$ consumption were observed to be lower in mitochondria of Dox animals compared with that of control and DE rats (Figure 4, A), we would normally anticipate $\mathrm{H}_{2} \mathrm{O}_{2}$ production in the Dox group to be proportionately low as well. Thus, elevation of $\mathrm{H}_{2} \mathrm{O}_{2}$ production in animals receiving doxorubicin to levels observed in control and $\mathrm{DE}$ groups indicates significant impairment in the efficiency of mitochondria of Dox animals to avoid free radical leakage from the respiratory chain (Figure 5, A).

This is best illustrated by calculation of the free radical leak (FRL\%), which is defined as the proportion of electrons that flow out of sequence in the electron transport chain (ETC) to reduce $\mathrm{O}_{2}$ to hydrogen peroxide instead of reaching cytochrome oxidase to reduce $\mathrm{O}_{2}$ to water. Because 2 electrons are needed to reduce 1 mole of $\mathrm{O}_{2}$ to hydrogen peroxide, whereas 4 electrons are required to 

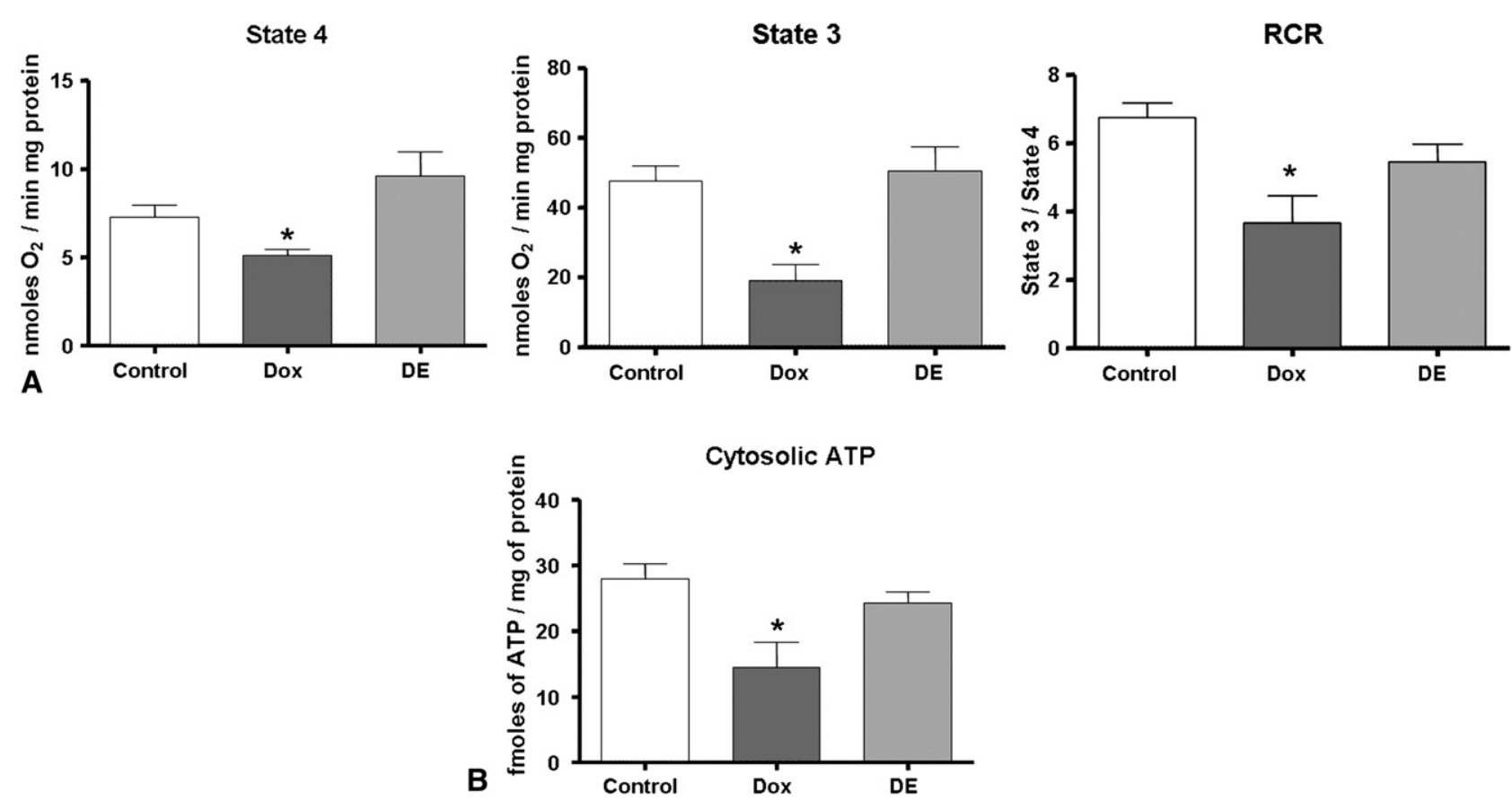

FIGURE 4. Enalapril prevents mitochondrial respiratory dysfunction and preserves cellular ATP content. A, We determined the effects of doxorubicin and enalapril on $\mathrm{O}_{2}$ consumption of isolated heart mitochondria. Oxygen consumption was measured during both states 4 and 3 via polarography using a Clarktype electrode. The RCR, an index of mitochondrial coupling and metabolic efficiency, was calculated by dividing state 3 to state 4 respiration values. Error bars represent SEM. *,\#P<.05. *Significantly different compared with DE group. \#Significantly different compared with control and DE groups. B, ATP content was measured in the cytosolic homogenate using an ATP bioluminescence kit. Cellular ATP content was found to be decreased in the Dox group compared with the control and DE groups. Error bars represent SEM. ${ }^{*} P<.05$. *Significantly different compared with control and DE groups. $R C R$, Respiratory control ratio; $A T P$, adenosine triphosphate; $D E$, doxorubicin + enalapril.

reduce 1 mole of $\mathrm{O}_{2}$ to water, the fraction of electrons that form ROS may be calculated by dividing the rate of $\mathrm{H}_{2} \mathrm{O}_{2}$ production by 2 times the rate of $\mathrm{O}_{2}$ consumption and multiplying the result by $100 .{ }^{18}$ Calculation of the FRL\% revealed a significant difference in ETC dysfunction between control and Dox animals (control: $2.29 \% \pm 0.27 \%$ vs Dox: $3.54 \% \pm 0.60 \%, P<.05$ ) (Figure $5, B$ ). Enalapril treatment abolished ETC dysfunction caused by doxorubicin (DE: $1.98 \% \pm 0.26 \%, P<.05$ vs Dox). These findings demonstrate that anthracycline administration causes leakage of free radicals, and that enalapril can ameliorate this negative effect.

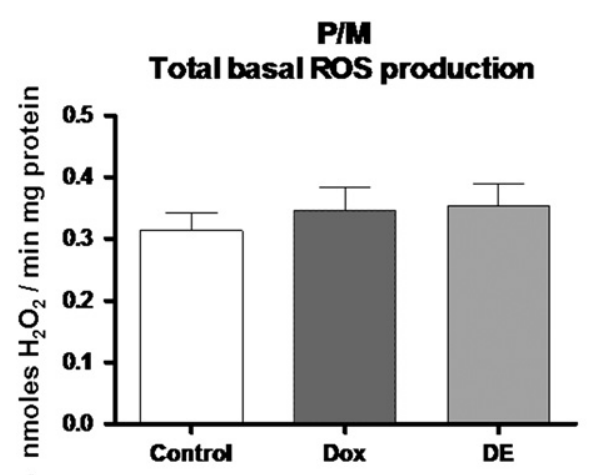

A

FIGURE 5. The increase in mitochondrial FRL, induced by doxorubicin, is completely abolished with simultaneous administration of enalapril. We measured $\mathrm{H}_{2} \mathrm{O}_{2}$ production in intact mitochondria of control, Dox, and DE animals. A, Heart mitochondria were obtained and supplemented with P/M as a substrate for oxidative phosphorylation. P/M was used to study total basal mitochondrial ROS production. B, The FRL \%, an index of mitochondrial efficiency, was calculated for $\mathrm{P} / \mathrm{M}$ supplemented mitochondria, by dividing the $\mathrm{H}_{2} \mathrm{O}_{2}$ values by twice the state 4 respiration values, and the result was multiplied by 100 to give a percent value. Error bars represent SEM. $* P<.05$. *Significantly different compared with control and DE groups. $P / M$, Pyruvate/malate; $F R L$, free radical leak; ROS, reactive oxygen species; Dox, doxorubicin; $D E$, doxorubicin + enalapril. 


\section{DISCUSSION}

The efficacy of doxorubicin as an anti-cancer drug has led to the search for diverse strategies to prevent its cytotoxic effects. ${ }^{19}$ Although many candidate compounds have been tested, surprisingly little research has examined the possible effect of ACE inhibitors on doxorubicin toxicity. Two recent clinical studies have demonstrated early treatment of anthracycline-induced cardiotoxicity with ACE inhibition significantly ameliorates development of heart failure. ${ }^{8,9}$ Although the authors of these studies do not present a definite mechanism for these results, they strongly postulate the antioxidant properties of enalapril are a primary mechanism by which ACE inhibition prevents against anthracycline cardiotoxicity. We demonstrate that enalapril does have a protective effect on mitochondrial function in doxorubicin-treated rats by (1) maintaining mitochondrial $\mathrm{O}_{2}$ consumption at control levels, (2) preventing the depletion of cellular ATP content, and (3) lowering the mitochondrial FRL.

It is important to recognize that treatment by ACE inhibition has been shown to have a number of beneficial effects on the cardiovascular system. These effects include reduction in mean arterial pressure, preload, and afterload, which contribute toward preservation of cardiac contractility. ${ }^{20}$ The systolic improvements observed in animals treated with enalapril in this study are almost certainly due in part to the beneficial effects of enalapril on the hemodynamic aspects of cardiac filling and output. The current study provides evidence for an additional mechanism of ACE inhibitors to ameliorate doxorubicin cardiotoxicity via the preservation of mitochondrial function and down-regulation of free radical generation. Rats treated with both enalapril and doxorubicin were observed to have higher levels of respiratory efficiency, minimal depletion of cytosolic ATP, and less oxidative stress as compared with animals receiving doxorubicin alone. Enalapril was able to attenuate the harmful effects of doxorubicin to near control levels in each of these respects and prevented deterioration in cardiac function and histopathologic grading.

Previous research has suggested that at the subcellular level, mitochondria may be a primary target of doxorubicin-induced cardiotoxicity. Specifically, it has been shown that doxorubicin-treated hearts exhibit mitochondrial abnormalities, including a decrease in respiratory efficiency, inhibition of electron transport complexes, and disruption of calcium homeostasis. ${ }^{21-23}$ In accordance with previous findings, we found that doxorubicin caused a robust decrease in state 3 respiration, which resulted in an overall robust decrease in the mitochondrial respiratory efficiency. The important observation is that enalapril prevented the decrease in state 4 and, more importantly, in state 3 oxygen consumption and RCR, thereby maintaining mitochondrial bioenergetics at normal levels. Similarly, we found that cellular ATP levels were significantly lowered after doxorubicin treatment, but that enalapril was able to maintain cellular ATP levels at near control values.

We did not detect any differences among groups in the amount of basal $\mathrm{H}_{2} \mathrm{O}_{2}$ produced and released by isolated mitochondria respiring with $\mathrm{P} / \mathrm{M}$ or succinate. However, these observations in combination with lower RCR and respiration rates of mitochondria isolated from Dox animals reinforced the hypothesis that doxorubicin induces ETC dysfunction (defined as the loss of ETC complex enzymatic activity or complex content). As support for these ideas, although basal $\mathrm{H}_{2} \mathrm{O}_{2}$ production was not different, the calculated FRL\% differed significantly among groups. Specifically, doxorubicin induced a significant increase in FRL $\%$, whereas enalapril completely abolished this effect, maintaining the FRL \% at control values. These results demonstrate that enalapril is capable of attenuating the fraction of electrons flowing out of sequence in the ETC, which ultimately reduce oxygen to form free radicals. In addition to causing cardiomyocyte dysfunction, generation of $\mathrm{O}_{2}^{-}$has also been linked to dysfunction of the coronary vasculature through inactivation of NO produced by endothelial cells and formation of $\mathrm{ONOO}^{-}$. Reduction in the bioavailability of NO is significant to the development of heart failure because the anti-inflammatory and vasodilation properties of NO are critical to maintaining cardiac function. ${ }^{24,25}$

\section{CONCLUSIONS}

This is the first study to assess the effects of ACE inhibitors on mitochondrial bioenergetics and recovery after chronic doxorubicin treatment. By maintaining the efficiency of mitochondrial respiration and cellular ATP content to near control levels, our results show that concurrent treatment with enalapril improves cardiac function in rats exposed to doxorubicin. Furthermore, the lowered potential of these mitochondria to produce ROS may contribute to the attenuation of dysfunction. Moreover, as has been suggested, ${ }^{15}$ we also found that cardiomyocyte apoptosis is only one contributing factor to the decreased cardiac function during doxorubicin cardiotoxicity. Last, these outcomes illustrate the importance of maintaining intact mitochondrial bioenergetics as a defense mechanism against doxorubicin cardiotoxicity, even in the case when part of the organ is lost or atrophied. Our findings, in conjunction with recent clinical reports that early treatment of enalapril after doxorubicin administration prevents development of heart failure, ${ }^{8,9}$ support a priori treatment of patients undergoing anthracycline chemotherapy with ACE inhibitors rather than after heart failure has been diagnosed.

\section{References}

1. Sacco G, Bigioni M, Evangelista S, Goso C, Manzini S, Maggi CA. Cardioprotective effects of zofenopril, a new angiotensin-converting enzyme inhibitor, on doxorubicin-induced cardiotoxicity in the rat. Eur J Pharmacol. 2001; 414:71-8. 
2. Lipshultz SE, Alvarez JA, Scully RE. Anthracycline associated cardiotoxicity in survivors of childhood cancer. Heart. 2008;94:525-33.

3. Zhou S, Starkov A, Froberg MK, Leino RL, Wallace KB. Cumulative and irreversible cardiac mitochondrial dysfunction induced by doxorubicin. Cancer Res. 2001;61:771-7.

4. Siveski-Iliskovic N, Kaul N, Singal PK. Probucol promotes endogenous antioxidants and provides protection against Adriamycin-induced cardiomyopathy in rats. Circulation. 1994;89:2829-35

5. Konorev EA, Kennedy MC, Kalyanaraman B. Cell-permeable superoxide dismutase and glutathione peroxidase mimetics afford superior protection against doxorubicin-induced cardiotoxicity: the role of reactive oxygen and nitrogen intermediates. Arch Biochem Biophys. 1999;368:421-8.

6. Pfeffer MA, Braunwald E, Moye LA, Basta L, Brown EJ Jr, Cuddy TE, et al. Effect of captopril on mortality and morbidity in patients with left ventricular dysfunction after myocardial infarction. Results of the survival and ventricular enlargement trial. The SAVE Investigators. N Engl J Med. 1992;327:669-77.

7. Boucek RJ Jr, Steele A, Miracle A, Atkinson J. Effects of angiotensin-converting enzyme inhibitor on delayed-onset doxorubicin-induced cardiotoxicity. Cardiovasc Toxicol. 2003;3:319-29.

8. Cardinale D, Colombo A, Sandri MT, Lamantia G, Colombo N, Civelli M, et al. Prevention of high-dose chemotherapy-induced cardiotoxicity in high-risk patients by angiotensin-converting enzyme inhibition. Circulation. 2006;114: 2474-81.

9. Cardinale D, Colombo A, Lamantia G, Colombo N, Civelli M, De Giacomi G. Anthracycline-induced cardiomyopathy: clinical relevance and response to pharmacologic therapy. J Am Coll Cardiol. 2010;55:213-20.

10. Tallaj JA, Franco V, Rayburn BK, Pinderski L, Benza RL, Pamboukian S, et al. Response of doxorubicin-induced cardiomyopathy to the current management strategy of heart failure. J Heart Lung Transplant. 2005;24:2196-201.

11. Richard C, Lauzier B, Delemasure S, Talbot S, Ghibu S, Collin B, et al. Effects of angiotensin-1 converting enzyme inhibition on oxidative stress and bradykinin receptor expression during doxorubicin-induced cardiomyopathy in rats. $J$ Cardiovasc Pharmacol. 2008;52:278-85.

12. Sanbe A, Tanonaka K, Kobayasi R, Takeo S. Effects of long-term therapy with ACE inhibitors, captopril, enalapril and trandolapril, on myocardial energy metabolism in rats with heart failure following myocardial infarction. $\mathrm{J} \mathrm{Mol} \mathrm{Cell}$ Cardiol. 1995;27:2209-22.
13. Barja G. The quantitative measurement of $\mathrm{H} 2 \mathrm{O} 2$ generation in isolated mitochondria. J Bioenerg Biomembr. 2002;34:227-33.

14. Abd El-Aziz MA, Othman AI, Amer M, El-Missiry MA. Potential protective role of angiotensin-converting enzyme inhibitors captopril and enalapril agains Adriamycin-induced acute cardiac and hepatic toxicity in rats. J Appl Toxicol. 2001;21:469-73

15. Zhu W, Soonpaa MH, Chen H, Shen W, Payne RM, Liechty EA, et al. Acute doxorubicin cardiotoxicity is associated with p53-induced inhibition of the mammalian target of rapamycin pathway. Circulation. 2009;119:99-106.

16. LaNoue K, Nicklas WJ, Williamson JR. Control of citric acid cycle activity in rat heart mitochondria. J Biol Chem. 1970;245:102-11.

17. Nojiri H, Shimizu T, Funakoshi M, Yamaguchi O, Zhou H, Kawakami S, et al Oxidative stress causes heart failure with impaired mitochondrial respiration. J Biol Chem. 2006;281:33789-801.

18. Sanz A, Caro P, Ibanez J, Gomez J, Gredilla R, Barja G. Dietary restriction at old age lowers mitochondrial oxygen radical production and leak at complex I and oxidative DNA damage in rat brain. J Bioenerg Biomembr. 2005;37:83-90.

19. Liu X, Chen Z, Chua CC, Ma YS, Youngberg GA, Hamdy R, Chua BH. Melatonin as an effective protector against doxorubicin-induced cardiotoxicity. Am J Physiol Heart Circ Physiol. 2002;283:H254-63.

20. Ader R, Chatterjee K, Ports T, Brundage B, Hiramatsu B, Parmley W. Immediate and sustained hemodynamic and clinical improvement in chronic heart failure by an oral angiotensin-converting enzyme inhibitor. Circulation. 1980;61:931-7.

21. Ascensao A, Magalhaes J, Soares JM, Ferreira R, Neuparth MJ, Marques F, et al. Moderate endurance training prevents doxorubicin-induced in vivo mitochondriopathy and reduces the development of cardiac apoptosis. Am J Physiol Heart Circ Physiol. 2005;289:H722-31.

22. Solem LE, Henry TR, Wallace KB. Disruption of mitochondrial calcium homeostasis following chronic doxorubicin administration. Toxicol Appl Pharmacol. 1994;129:214-22.

23. Wallace KB. Doxorubicin-induced cardiac mitochondrionopathy. Pharmacol Toxicol. 2003;93:105-15.

24. Davidson SM, Duchen MR. Endothelial mitochondria: contributing to vascular function and disease. Circ Res. 2007;100:1128-41.

25. Doughan AK, Harrison DG, Dikalov SI. Molecular mechanisms of angiotensin II-mediated mitochondrial dysfunction: linking mitochondrial oxidative damage and vascular endothelial dysfunction. Circ Res. 2008;102:488-96. 


\section{SUPPLEMENTAL MATERIALS AND METHODS Animals}

Twenty-four female Sprague Dawley rats at approximately 10 weeks of age were obtained from Charles River Laboratories (Wilmington, Mass). The animals were divided into 3 groups: (1) control-untreated $(n=8),(2)$ doxorubicin treated (Dox) $(\mathrm{n}=8)$, and (3) Dox + enalapril treated (DE) $(\mathrm{n}=8)$ (Figure 1, $A$ ). Both Dox and DE groups received doxorubicin at a cumulative dose of $25 \mathrm{mg} / \mathrm{kg}$ administered weekly via intraperitoneal injection for 6 weeks. Enalapril pretreatment for the DE group was started 1 week before administration of the first doxorubicin injection and continued throughout the study and for an additional 3 weeks after the last doxorubicin injection. Enalapril was administered in the drinking water at a dose of $10 \mathrm{mg} / \mathrm{kg} / \mathrm{d}$ for each animal as described in previous studies. ${ }^{\mathrm{E} 1}$ Body weights were recorded at 2 time points: at 5 weeks from enalapril administration (midpoint) and at 10 weeks (end point). Hearts were explanted and weighed at the end point (Figure E1).

\section{Assessment of Left Ventricular Fractional Shortening}

For echocardiographic measurement of ventricular function, animals were knocked down using $1 \%$ isoflurane and anesthesia was maintained with inhaled isoflurane (1\%). Echocardiographic measures were conducted on a short-axis plane, and M-mode was used for the final measurements of fractional shortening. The mean heart rate was measured during the procedure. Baseline echocardiograms were conducted in all animals before treatment. Echocardiography studies were performed at baseline before treatment with doxorubicin and immediately before animal sacrifice, using a $14.7-\mathrm{MHz}$ transducer on a Sequoia C512 echocardiography system (Siemens, Malvern, Pa). The left ventricular end-diastolic diameter and end-systolic diameter were measured by a blinded investigator and processed to calculate left ventricular fractional shortening by the formula: LVFS $=[$ EDD-ESD $] / E D D$, where LVFS is left ventricular fractional shortening, EDD is end-diastolic diameter, and ESD is end-systolic diameter.

\section{Postmortem Histology}

At the time of death, a subset of the animal hearts and livers was fixed in $4 \%$ paraformaldehyde, subsequently cut, and embedded in paraffin blocks. The blocks were then sectioned into short-axis slices, which were subsequently stained with hematoxylin-eosin stain according to standard protocols. High-power fields of the sections were photographed.

\section{Mitochondrial and Cytosolic Isolation}

Mitochondrial and cytosolic protein fractions were isolated using differential centrifugation. Specifically, at the end of the study period, animals were anesthetized using isoflurane gas. When a general plane of anesthesia was reached, the chest was opened and the heart was excised, cleaned with phosphate-buffered saline, and weighed. The heart was finely minced into small pieces and homogenized in $(1: 8 \mathrm{wt} / \mathrm{vol})$ ice-cold isolation buffer containing $220 \mathrm{mmol} / \mathrm{L}$ mannitol, $70 \mathrm{mmol} / \mathrm{L}$ sucrose, $1 \mathrm{mmol} / \mathrm{L}$ EDTA, 10 $\mathrm{mmol} / \mathrm{L}$ Tris, and $0.2 \%$ fatty acid free bovine serum albumin, $\mathrm{pH} 7.4$, using a Potter-Elvehjem glass homogenizer (Glas-Col LLC, Terre Haute, Ind). The homogenate was centrifuged at $700 \mathrm{~g}$ for 10 minutes. Part of the supernatant was used immediately for the measurement of ATP content, and the remaining supernatant was then centrifuged at $8000 \mathrm{~g}$ for 10 minutes. The supernatant (crude cytosol) was stored at $-80^{\circ} \mathrm{C}$ for further analysis, and the mitochondrial pellet was resuspended in $200 \mu \mathrm{L}$ of isolation buffer without bovine serum albumin and used immediately for the measurements of mitochondrial $\mathrm{H}_{2} \mathrm{O}_{2}$ production and oxygen consumption. All centrifugation steps were carried out at $4{ }^{\circ} \mathrm{C}$. Protein concentrations were determined using the Bradford method. ${ }^{\mathrm{E} 2}$

\section{Enzymatic Measurement of Caspase-3 and Caspase-9 Activities}

Heart cytosolic fractions were prepared and caspase- 3 and caspase- 9 activities were measured using fluorometric protease assay kits: Caspase3/CPP32 and Caspase-9/Mch6 (Biovision, Mountain View, Calif) according to the manufacturer's instructions. Briefly, in these assays, the substrates DEVD-AFC and LEHD-AFC are cleaved by caspase- 3 and -9 respectively, releasing free AFC emitting a yellow-green fluorescence $(\lambda$ $\max =505 \mathrm{~nm}$ ) that is quantified using a fluorescence microtiter plate reader. Samples were run in triplicate, and values were expressed as raw fluorescence units per milligram of cytosolic protein.

\section{Mitochondrial Respiration}

Mitochondrial oxygen consumption was measured at $37^{\circ} \mathrm{C}$ by polarography, with a Clark-type oxygen electrode (Oxytherm, Hansatech, Norfolk, UK) under identical conditions (same mitochondria, buffer composition, and substrate concentrations) to $\mathrm{H}_{2} \mathrm{O}_{2}$ production measurements: incubation buffer $(145 \mathrm{mmol} / \mathrm{L} \mathrm{KCl}, 30 \mathrm{mmol} / \mathrm{L}$ Hepes, $5 \mathrm{mmol} / \mathrm{L} \mathrm{KH} 2 \mathrm{PO} 4,3 \mathrm{mmol} / \mathrm{L}$ $\mathrm{MgCl} 2,0.1 \mathrm{mmol} / \mathrm{L}$ EGTA, $\mathrm{pH}$ 7.4) with $1 \mathrm{mg}$ of mitochondrial protein per milliliter and $2.5 \mathrm{mmol} / \mathrm{L}$ pyruvate $/ 2.5 \mathrm{mmol} / \mathrm{L}$ malate as substrates. The assay was performed in the absence (state 4 : resting state) and presence (state 3 : phosphorylating state) of $500 \mu \mathrm{mol} / \mathrm{L}$ ADP. Clark-type electrode without (state 4) and with saturant ADP (state 3) allows calculation of the RCR (state $3 /$ state 4 oxygen consumption) as an indicator of the degree of coupling and metabolic activity of the mitochondrial preparations.

\section{Adenosine Triphosphate Content}

Mitochondria and homogenate isolated from heart were used immediately after isolation to determine ATP content using a bioluminescence ATP kit from Sigma-Aldrich Inc (St Louis, sMO), following the manufacturer's protocol. This bioluminescence assay is based on the reaction of ATP with recombinant firefly luciferase and its substrate D-Luciferin. On addition, ATP combines with Luciferin to form luciferyl adenylate and inorganic pyrophosphate on the surface of the luciferase enzyme. While bound to the enzyme, luciferyl adenylate combines with $\mathrm{O}_{2}$ to form oxyluciferin and AMP through a series of enzymatic redox reactions. As oxyluciferin and AMP are released from the enzyme's surface, a quantum yield of light is emitted in proportion to the ATP concentration in the sample. The light emission can then be recorded and quantified using a chemiluminometer. Mitochondria and homogenates were suspended in a lysis buffer for 3 minutes, followed by the addition of the reaction mixture containing luciferase and luciferin. ATP content was measured with a Packard LumiCount luminometer (Packard, United Kingdom), using an ATP standard curve. Final mitochondrial and homogenate concentrations used in the assay were $1 \mathrm{mg} / \mathrm{mL}$.

\section{Mitochondrial $\mathrm{H}_{2} \mathrm{O}_{2}$ Generation}

The rate of mitochondrial $\mathrm{H}_{2} \mathrm{O}_{2}$ production was assayed in freshly isolated mitochondria by a highly sensitive fluorometric method according to $\mathrm{Barja}^{\mathrm{E} 3}$ and adapted to a microplate reader. $\mathrm{H}_{2} \mathrm{O}_{2}$ generation was monitored by measuring the increase in fluorescence (excitation at $312 \mathrm{~nm}$, emission at $420 \mathrm{~nm}$ ) due to the oxidation of homovanillic acid by $\mathrm{H}_{2} \mathrm{O}_{2}$ in the presence of horseradish peroxidase. The assay was performed in incubation buffer $(145 \mathrm{mmol} / \mathrm{L} \mathrm{KCl}, 30 \mathrm{~mol} / \mathrm{L}$ Hepes, $5 \mathrm{mmol} / \mathrm{L} \mathrm{KH} 2 \mathrm{PO} 4,3 \mathrm{mmol} / \mathrm{L}$ $\mathrm{MgCl} 2,0.1 \mathrm{mmol} / \mathrm{L}$ EGTA, $0.2 \%$ bovine serum albumin, $\mathrm{pH} 7.4)$ at $37^{\circ} \mathrm{C}$, and the reaction conditions were $0.25 \mathrm{mg}$ of mitochondrial protein per $\mathrm{mL}, 6 \mathrm{U} / \mathrm{mL}$ of horseradish peroxidase, and $0.1 \mathrm{mmol} / \mathrm{L}$ homovanillic acid. The reaction was started by the addition of $2.5 \mathrm{mmol} / \mathrm{L}$ pyruvate/ $2.5 \mathrm{mmol} / \mathrm{L}$ malate, or $5 \mathrm{mmol} / \mathrm{L}$ succinate as substrates. $\mathrm{P} / \mathrm{M}$ was used to study total basal ROS production, and succinate was used to study complex III ROS production under basal conditions as described previously. ${ }^{\mathrm{E} 4}$ After 15 minutes of incubation at $37^{\circ} \mathrm{C}$, the reaction was stopped and the samples were transferred on ice and a stop solution $(0.1 \mathrm{~mol} / \mathrm{L}$ glycine, $25 \mathrm{mmol} / \mathrm{L}$ 
EDTA, pH 12) was added. Known amounts of $\mathrm{H}_{2} \mathrm{O}_{2}$ generated in parallel by glucose oxidase, with glucose as substrate, were used as standards. We also used inhibitors of the ETC to study maximum rates of $\mathrm{H}_{2} \mathrm{O}_{2}$ production from complexes I and III, because they represent the main sites of ROS generation (especially complex I) within the mitochondria. For complex I maximum rate, we used $2 \mu \mathrm{mol} / \mathrm{L}$ rotenone added to $\mathrm{P} / \mathrm{M}$-supplemented mitochondria. For complex III maximum rate, we used $2 \mu \mathrm{mol} / \mathrm{L}$ antimycin A plus $2 \mu \mathrm{mol} /$ $\mathrm{L}$ rotenone added to succinate-supplemented mitochondria. In addition, the assays with succinate as substrate were performed in the presence of $2 \mu \mathrm{mol} /$ L rotenone to avoid the backward flow of electrons to complex I. All samples were run in duplicate. $\mathrm{H}_{2} \mathrm{O}_{2}$ production and $\mathrm{O}_{2}$ consumption were measured in parallel in the same heart mitochondria under similar experimental conditions. This allowed the calculation of the fraction of electrons out of sequence that reduce $\mathrm{O}_{2}$ to ROS at the respiratory chain (FRL\%) instead of reaching cytochrome oxidase to reduce $\mathrm{O}_{2}$ to water. Because 2 electrons are needed to reduce 1 mole of $\mathrm{O}_{2}$ to $\mathrm{H}_{2} \mathrm{O}_{2}$ and 4 electrons are transferred in the reduction of 1 mole of $\mathrm{O}_{2}$ to water, the $\mathrm{FRL} \%$ can be calculated as the rate of $\mathrm{H}_{2} \mathrm{O}_{2}$ production divided by 2 times the rate of $\mathrm{O}_{2}$ consumption, and the result is multiplied by 100 .

\section{SUPPLEMENTAL RESULTS}

\section{Animal Survival, Body Weight, and Heart Mass}

There were no deaths in the control and DE groups $(100 \%$ survival) during the experimental protocol. However, 2 rats from the Dox group died of heart failure during weeks 9 and 10 (75\% survival; log-rank Mantel-Cox test: $P=.11$; NS compared with control and DE; Figure $1, B$ ). Body weights were recorded at 2 time points: at 5 weeks into the study (midpoint) and at 10 weeks (end point) (Figure E1, A). Heart weights were recorded immediately after heart extraction at end point (Figure E1, B). There was a significant decrease in body weight in both the Dox $(237.1 \pm 6.7 \mathrm{~g})$ and $\mathrm{DE}(215.8 \pm 5.5 \mathrm{~g})$ groups compared with the control rats $(278.1 \pm 10.2 \mathrm{~g})$ at week 5 (both $P<.05$; Figure E1, $B)$. By week 10 , total body weight decreased for both the Dox $(196.9 \pm 11.9 \mathrm{~g})$ and DE $(208 \pm$ $9.73 \mathrm{~g})$ groups compared with control rats $(287.8 \pm 12.64$ g) (both $P<.05)$. Heart weight diminished significantly in the Dox $(0.52 \pm 0.03 \mathrm{~g})$ and $\mathrm{DE}(0.52 \pm 0.025 \mathrm{~g})$ groups compared with control $(0.78 \pm 0.019 \mathrm{~g})$ at week 10 (both $P<.05$; Figure E1, $B$ ), indicative of heart damage and possible cardiomyocyte apoptosis. It is evident that doxorubicin treatment resulted in significant body mass and heart mass losses that were not prevented by enalapril administration. As expected, the body weight to heart weight ratio did not differ significantly among the groups (control: $0.0028 \pm$ 0.0001 vs Dox: $0.0027 \pm 0.0001$ vs DE: $0.0025 \pm 6.230$ ) because both heart weight and body weight were decreased by doxorubicin to the same proportion, resulting in stable ratios.

\section{Baseline Echocardiographic Measures and Heart Rate for Assessment of Cardiac Function}

The mean heart rate for all animals during the echocardiographic procedure was $410 \pm 20$ beats/min at baseline before treatment with doxorubicin and $405 \pm 15$ beats/ min at the study end point $(P=\mathrm{NS})$. There was no statistical difference between the control DOX and DE groups. In addition, fractional shortening values calculated at baseline were not significantly different from the control group's values at study end (baseline of all animals before Dox treatment: $38.62 \pm 0.77$, control group at study end: $39.28 \pm 0.83)$.

\section{Supplemental References}

E1. Sanbe A, Tanonaka K, Kobayasi R, Takeo S. Effects of long-term therapy with ACE inhibitors, captopril, enalapril and trandolapril, on myocardial energy metabolism in rats with heart failure following myocardial infarction. $J \mathrm{Mol}$ Cell Cardiol. 1995;27:2209-22.

E2. Bradford MM. A rapid and sensitive method for the quantitation of microgram quantities of protein utilizing the principle of protein-dye binding. Anal Biochem. 1976;72:248-54.

E3. Barja G. The quantitative measurement of $\mathrm{H} 2 \mathrm{O} 2$ generation in isolated mitochondria. J Bioenerg Biomembr. 2002;34:227-33.

E4. Barja G. Mitochondrial oxygen radical generation and leak: sites of production in states 4 and 3, organ specificity, and relation to aging and longevity. J Bioenerg Biomembr. 1999;31:347-66. 

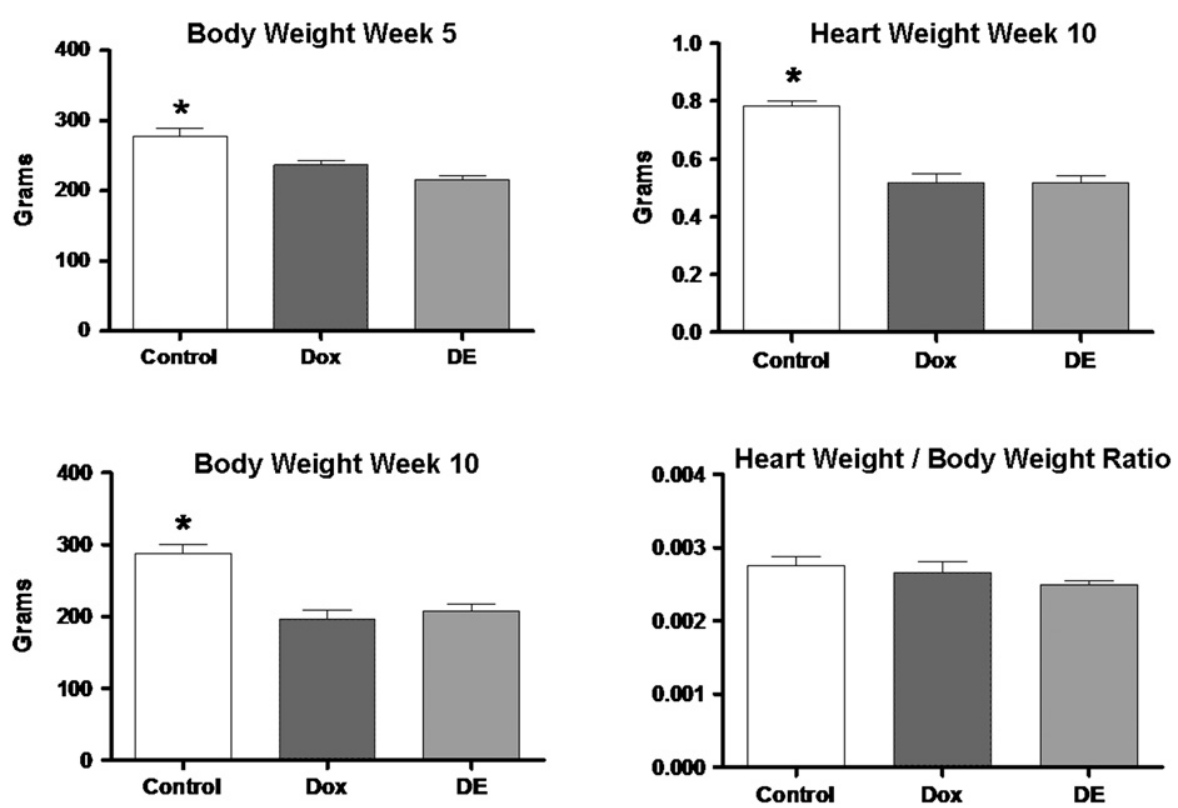

FIGURE E1. Doxorubicin treatment induces a significant loss of body mass and heart mass in both Dox and DE groups. A, Body weights were recorded at 5 weeks into the study (midpoint) and at 10 weeks (end point). B, Heart weights were recorded on heart extraction at end point. The heart weight to total body weight ratio was also calculated. Error bars represent SEM. $* P<.05$. *Significantly different compared with Dox and DE groups. Dox, Doxorubicin; $D E$, doxorubicin + enalapril. 